\title{
The Effects of the Sub-carrier Grouping on Multi-Carrier Channel Aware Scheduling *
}

\author{
Fanchun Jin, Gokhan Sahin, Amrinder Arora, and Hyeong-Ah $\mathrm{Choi}^{\dagger}$
}

\begin{abstract}
Channel-aware scheduling and link adaptation (LA) methods are widely considered to be crucial for realizing high data rates in wireless networks. Multi-carrier systems that spread information bits over the entire signal band can take advantage of the frequency selective fading, and choose the sub-carrier(s) that have the best channel conditions for transmission. However, predicting the future channel states, and adjusting the transmission schedules and parameters accordingly, may consume valuable system resources, such as bandwidth, time, and power. Sub-carrier grouping, which refers to monitoring/treating a set of adjacent sub-carriers as a single unit, has been proposed to reduce the overhead associated with LA. This paper considers various models for sub-carrier grouping, each representing a different level of system complexity, and investigates the trade-offs between the sub-carrier grouping granularity and the link throughput. We first present an offline dynamic programming algorithm for finding an optimal solution, assuming perfect estimates of the channel states. The solution obtained through this formulation can serve as a performance bound for online algorithms. We then propose an online algorithm for the case of perfect channel state estimates, which is shown to have very close performance to the off-line optimal solution. This online algorithm is also extended to incorporate imperfect channel state estimates due to (i) variations in channel states among the sub-carriers within the same group, and (ii) prediction inaccuracies in estimating the future time-slots. Performances of the algorithms are evaluated through simulations and comparisons with the off-line solution. The trade-off between the sub-carrier grouping granularity and the link throughput is also presented. Overall, our results can provide some guidelines from the network scheduling perspective for deciding what kind of sub-carrier grouping model and granularity to use in multi-carrier networks with LA.
\end{abstract}

\section{Introduction}

Multi-carrier wireless systems have become a promising means of achieving the high data rates required by emerging wireless applications, especially when combined with link adaptation. Link Adaptation (LA) loosely refers to changing

\footnotetext{
${ }^{*}$ This work was supported in part by U.S. Army Research Offi ce under contract DAAD 19-02-D-001, TCN 02-007

${ }^{\dagger}$ F. Jin, A. Arora and H.-A. Choi are with the Dept. of Computer Science and G. Sahin is with the Dept. of ECE, George Washington University, Washington, DC 20052. e-mail: \{jinfc,gokhan,amrinder,hchoi\}@gwu.edu.
} 
transmission parameters over a link, such as modulation, coding rate, power, etc., in response to changing channel conditions. The adaptation of the transmission parameters is performed according to the predicted future quality of the channel, also called as the channel-state. We assume that each channel (sub-carrier) may be in one of $M$ different states at any given time, with each state corresponding to a different pre-determined data rate achievable through LA. The presence of multiple carriers, combined with LA, leads to the possibility of choosing which sub-carriers to use, and at what rate to transmit on each sub-carrier in each time-slot.

While it is desirable to adapt the transmission parameters according to the channel state information (CSI) to capture even small-scale variations, there are practical limitations to frequent link adaptation. Fast adaptation increases the number of mode-change messages transmitted over the channel, consuming bandwidth, and time resources [1]. While many aspects of scheduling transmissions over time-varying wireless channels have been studied (see, for example, $[2,3,4]$ and the references therein), the penalty induced by LA has not been considered. Moreover, predicting the future channel quality may also consume signifi cant amount of system resources (e.g., time, bandwidth and power), since it may involve transmission of training-sequences, pilot tones, or feedback messages carrying the CSI. The issue of predicting the channel states and updating transmission modes becomes even more serious when considered within the context of multiple carriers if each sub-carrier is individually tracked/adapted.

One approach to reduce the overhead associated with LA in multi-carrier systems is sub-carrier grouping, i.e., forming groups of sub-carriers consisting of neighboring sub-carriers, and treating the sub-carriers within the group as a whole for LA. For example, the sub-carriers within the same group may be constrained to adjust their rates in exactly the same time slot, or even to use the same data rates, resulting in a reduction in the number of modechange messages. Further savings in system resources can be obtained through coarser CSI monitoring techniques, e.g., by using CSI of the center sub-carrier in each group as the representative of the whole group [5]. Clearly, these additional constraints may reduce the overall throughput, but they may also bring advantages by alleviating the additional resource consumption associated with LA.

This paper studies the effects of the sub-carrier grouping on the scheduling problem in a wireless network with multiple carriers. In particular we consider the trade-offs between size of the sub-carrier groups and the link throughput for various sub-carrier grouping models, with each corresponding to a different level of CSI tracking and system design complexity. We assume that a single user is communicating with the base station using the entire sub-carriers. This assumption is motivated by the OFDM, which uses multiple carriers in order to benefi t from the frequency selective fading introduced by spreading the information bits over the entire signal band $[1,5]$. The performance can 
potentially be improved by using high-level modulation on the sub-carriers with good channel conditions, and avoiding transmission on those with bad channel conditions. Ideally, each sub-carrier may use a different coding and modulation scheme (mode) independently of the others. However, practical limitations, such as the overhead involved in tracking CSI for each sub-carrier, as well as implementation complexity issues may favor other design options. For example, many implementations of OFDM involve joint processing of the signals on different sub-carriers at the transmitter and the receiver $[6,7,5]$, in order to simplify the implementation over a design that associates an independent transmitter and receiver with each sub-carrier. The model considered in this paper also differs from earlier work on wireless network scheduling in that we explicitly consider the time-penalty associated with link adaptation. In particular, we consider a wireless system with closed-loop adapation [5], where the changes in the transmission mode need to be explicitly signaled from the receiver to the transmitter, resulting in a time delay in starting transmission with the new data rates.

Four sub-carrier grouping models are considered in this paper, depending on whether: (i) CSI for each sub-carrier is known perfectly or not at the beginning of the current time-slot, and (ii) CSI for each sub-carrier in the same group is monitored individually or not. If CSI for each sub-carrier is not individually monitored, then it is assumed, without loss of generality, that only the sub-carrier located in the center of each group is monitored/tracked in order to reduce the overhead associated with the tracking. We will refer to the two conditions mentioned above which are used to categorize different cases as time-based estimation accuracy, and spectrum-based estimation accuracy, respectively, since the first one deals with whether our estimates are accurate over time, and the second one deals with whether our estimates are accurate over the frequency range of the group. Furthermore, for each case we consider, the rate allocation for all sub-carriers in the same group may be either constrained to be the same or allowed to be different. Assigning the same mode (data rate) to all sub-carriers within a group is mainly motivated by reducing the overhead involved in CSI tracking and mode-change messages [5, 1]. Even when the use of different modes is allowed within the same group, the implementation may be simplifi ed by synchronizing the mode-changes among the sub-carriers within the same group, since many OFDM implementations involve joint processing of the signals transmitted on different sub-carriers as discussed above.

The rest of the paper is organized as follows. In Section 2, we discuss the system model and notations considered in this paper and give a formal statement of our problem. An offline dynamic programming algorithm for fi nding an optimal solution for the case of spectrum- and time-perfect estimates (with the same or different rate allocation requirement) is presented in Section 3. This optimal algorithm will be used as an upper bound in later sections for 
throughput analysis. In Section 4, an online algorithm for the case with perfect CSI estimates in spectrum and time is discussed. Heuristics for online variations considering spectrum- and time-estimation accuracy probabilities are also discussed in Section 4. Numerical results are presented in Section 5, and the paper concludes in Section 6.

\section{Problem Statement}

We consider the downlink scheduling problem in a wireless network with 1 user and $f$ sub-carriers $\beta_{1}, \beta_{2}, \ldots, \beta_{f}$. Note that we use the terms channel and sub-carrier interchangeably in this paper. Time is slotted, and time-divisionduplexing is used, i.e., uplink and downlink transmissions take place in turn. At any given timeslot $t$, the maximum data rate for the downlink on sub-carrier $j$ is bounded by $c(j, t)$. If the base station tries to transmit data at the rate larger than $c(j, t)$, the actual throughput on sub-carrier $j$ in timeslot $t$ is 0 .

We divide sub-carriers $\beta_{1}, \beta_{2}, \ldots, \beta_{f}$ to $m$ groups, such that index-continuous $k$ sub-carriers are in each group, except posssibly for the last group (in case $f$ is not divisible by $k$ ).

$$
\begin{aligned}
G_{1} & =\left\{\beta_{1}, \beta_{2}, \ldots, \beta_{k}\right\} \\
G_{2} & =\left\{\beta_{k+1}, \beta_{k+2}, \ldots, \beta_{2 k}\right\} \\
& \vdots \\
G_{m} & =\left\{\beta_{(m-1) k+1}, \beta_{(m-1) k+2}, \ldots, \beta_{f}\right\}
\end{aligned}
$$

Please note that $m=\lceil f / k\rceil$. In each group, the base station performs link adaptation on the basis of group rather than individual sub-carrier, i.e., link adaptations for all sub-carriers within the same group are always performed in the same timeslot. Again, the base station can allocate a transmission rate which is larger than the current capacity for a sub-carrier $\beta_{j}$ in timeslot $t$, and, if that is the case, the actual throughput, denoted by $x(j, t)$, for the sub-carrier in timeslot $t$ is 0 .

We assume that the base station has perfect knowledge of the channel state for the sub-carrier located in the center (based on index) of a group in timeslot $t$, and perfect or imperfect estimates of the channel states for the remaining sub-carriers in that group. We also assume that the base station has perfect or imperfect estimates of channel states for the next timeslot $t+1$. The base station allocates a transmission rate for each channel in timeslot $t$ according to the previous timeslot allocations for each sub-carrier in the group, as well as the current and next time-slot capacity estimates of all sub-carriers in the group. If an allocation change is required for a sub-carrier, then the next downlink transmission (in time-slot $t$ ) is used to notify the receiver of the new transmission rate, which may be confi rmed by the 
mobile station through an ACK in the next uplink transmission. Thus, transmission at the new data rate starts only after a delay of a full duplex transmission cycle. Let us assume that each time-slot, of duration $\delta$, consists of a downlink transmission followed by an uplink transmission. Thus, if the base station decides to change the transmission rate at the beginning of time slot $t \delta$, then transmission with the new rate starts at the beginning of time slot $(t+1) \delta$. The duration of each timeslot is assumed to be equivalent to this time-penalty $\delta$. Hence, the earliest time to have a new data rate after timeslot $t$ is $t+2$. Since we consider group-wise link adaptation, if link adaptation needs to be performed for one channel, all other channels in the same group also have to pay the penalty of link adaptation. That is, if the allocated transmission rate $a(j, t)$ for sub-carrier $\beta_{j}$ in $t$ th timeslot is different from allocation in $(t+2)$ th timeslot, then allocation during timeslot $(t+1)$ must be 0 for all channels that are in the same group as $\beta_{j}$.

$$
\text { If } a(j, t) \neq a(j, t+2) \text {, then } a(i, t+1)=0 \forall i: \beta_{i} \in G\left(\beta_{j}\right)
$$

An example of feasible allocation matrix for a group with size of 3 and 6 timeslots is shown in Table 1

Table 1: Feasible allocation matrix for a group with size 3 and 6 timeslots.

\begin{tabular}{|c||c|c|c|c|c|c|}
\hline & $\mathrm{t} 1$ & $\mathrm{t} 2$ & $\mathrm{t} 3$ & $\mathrm{t} 4$ & $\mathrm{t} 5$ & $\mathrm{t} 6$ \\
\hline \hline$\beta 1$ & 10 & 10 & 0 & 11 & 0 & 20 \\
\hline$\beta 2$ & 11 & 11 & 0 & 10 & 0 & 10 \\
\hline$\beta 3$ & 12 & 12 & 0 & 12 & 0 & 12 \\
\hline
\end{tabular}

\subsection{Perfect and Imperfect Estimates}

We explore four cases of the scheduling problem according to the knowledge of the base station about the channel states for the sub-carriers other than the center sub-carrier of a group and the estimated next timeslot capacities. For each case, we consider two sub-cases. First sub-case is that the allocation for all sub-carriers in the same group is the same, i.e., $a(i, t)=a(j, t)$ if $\beta_{i}$ and $\beta_{j}$ are in the same group. The second sub-case is that the allocation for each sub-carrier in the same group can be different.

- Case 1: Time based perfect estimates and spectrum based perfect estimates(TPSP). The estimated capacities for next timeslot is assumed to be perfect and the channels in the group are monitored separately, so that the current capacity estimate for each channel is perfect.

- Case 2: Time based imperfect estimates and spectrum based perfect estimates(TISP). The estimated capacities for next timeslot is assumed to be imperfect so that the actual capacity for next timeslot may be different 
from the estimated capacity. The channels in the group are monitored separately, so that current capacity estimate for each channel is perfect.

- Case 3: Time based perfect estimates and spectrum based imperfect estimates(TPSI). The estimated capacities for next timeslot is assumed to be perfect. However, only the center channel of a group is monitored and the other channels in that group have imperfect capacity estimates for the current timeslot.

- Case 4: Time based imperfect estimates and spectrum based imperfect estimates(TISI). The estimated capacities for next timeslot is assumed to be imperfect and only the channel which is located in the center of a group is monitored, so that the capacities for remaining channels in that group are estimated imperfectly.

Our objective is to develop online and/or offline algorithms and to fi nd the relationship between the overall achievable throughput, $\sum_{j=1}^{f} \sum_{t=1}^{n} x(j, t)$, and the grouping size, $k$.

\subsection{Notation}

The following notations are used in this paper repetitively.

- $f$ : Total number of subchannels

- $k$ : The number of subchannels in one group

- $m$ : The number of groups

- The channels are assumed to be grouped as follows:

$$
\begin{aligned}
& G_{1}=\left\{\beta_{1}, \beta_{2}, \beta_{3}, \ldots \beta_{k}\right\} \\
& G_{2}=\left\{\beta_{k+1}, \beta_{k+2}, \beta_{k+3}, \ldots, \beta_{2 k}\right\} \\
& \vdots \\
& G_{m}=\left\{\beta_{(m-1) k+1}, \beta_{(m-1) k+2}, \ldots, \beta_{f}\right\}
\end{aligned}
$$

- $G\left(\beta_{i}\right)$ : Group that channel $\beta_{j}$ belongs in.

- $\beta_{j}$ : The $j$ th subchannel, where $1 \leq j \leq f$

- $c(j, t)$ : Capacity available on subchannel $\beta_{j}$ during the $t$ th timeslot

- $c^{\prime}(j, t+1)$ : Value of $c(j, t+1)$, as estimated in timeslot $t$ (time based estimation) 
- $c_{j}^{*}(i, t)$ : Value of $c(i, t)$, as estimated by monitoring $\beta_{j}$ (spectrum based estimation). It is assumed that the base station has perfect knowledge of $c(j, t)$ at timeslot $t$.

- $a(j, t)$ : Allocation for subchannel $\beta_{j}$ at $t$ th timeslot.

- $\hat{a}(j, t+1)$ : Tentative allocation for subchannel $\beta_{j}$ at $(t+1)$ th timeslot.

- $x(j, t)$ : Actual throughput for subchannel $\beta_{j}$ at $t$ th timeslot.

- $\hat{x}(j, t+1)$ : Tentative throughput for subchannel $\beta_{j}$ at $(t+1)$ th timeslot.

$$
x(j, t)= \begin{cases}a(j, t) & \text { if } a(j, t) \leq c(j, t) \\ 0 & \text { otherwise }\end{cases}
$$

- $P(a, b, j, t+1)$ : Probability that actual capacity $c(j, t+1)=b$ given estimated $c^{\prime}(j, t+1)=a$.

- $Q_{j}(a, b, i, t)$ : Probability that actual capacity $c(i, t)=b$ given estimated $c_{j}^{*}(i, t)=a$.

\section{Optimal Offline Solution given Perfect Estimates}

In this section, we present a dynamic programming algorithm for fi nding an optimum solution for the offline version of the problem. We assume that the channel capacity for all timeslots and all subcarriers is known, and the grouping is specifi ed. Our objective is to allocate usage so as to maximize the total throughput.

Clearly, each group is independent of the others, and thus, we will formulate the problem as maximization problem for the specifi ed group only.

Given: A group of sub-carriers $\beta_{1}, \beta_{2}, \ldots \beta_{k}$, and capacities $c(j, t), 1 \leq j \leq k, 1 \leq t \leq n$.

To Do: Allocate $a(j, t), 1 \leq j \leq k, 1 \leq t \leq n$.

Objective: Maximize $\sum_{j=1}^{k} \sum_{t=1}^{n} x(j, t)$, where $x(j, t)=a(j, t)$ if $a(j, t) \leq c(j, t)$ and $x(j, t)=0$ otherwise.

Before giving a solution to the problem, we make the following observation, which is critical in defining the recurrance relation.

Observation 1: For any feasible schedule for any timeslots between $t_{1}$ and $t_{2}$, one of the cases must hold:

- Case I: There exists a timeslot, say $t^{\prime}$, for which channel allocation is 0 for all channels.

- Case II: For each sub-carrier, channel allocation will remain constant over all timeslots. 


\subsection{Recurrance}

In this case the recurrance is given as follows.

$$
A\left(t_{1}, t_{2}\right)=\max \begin{cases}\max _{t_{1} \leq t^{\prime} \leq t_{2}}\left\{A\left(t_{1}, t^{\prime}-1\right)+A\left(t^{\prime}+1, t_{2}\right)\right\} & \text { (Case I holds) } \\ \left.\sum_{1 \leq j \leq k} B\left(j, t_{1}, t_{2}\right)\right\} & \text { (Case II holds) }\end{cases}
$$

Here, $B\left(j, t_{1}, t_{2}\right)$ is the maximum throughput on channel $j$ without using link adaptation between timeslots $t_{1}$ and $t_{2}$, that is, by using same allocation on each timeslot between $t_{1}$ and $t_{2}$. We observe, that even though the allocation on each timeslot will be the same, the throughput as given by Equation 1 need not be the same. For calculating the value of $B\left(j, t_{1}, t_{2}\right)$, we consider two models:

- For a given timeslot, all channels must have the same allocation.

- For a given timeslot, different channels can have different allocation.

We observe that $B\left(j, t_{1}, t_{2}\right)$ is the product of allocation and the number of timeslots for which the capacity matches or exceeds that value of allocation. If a high value of allocation is chosen, few timeslots may be able to utilize that allocation. If a low value of allocation is chosen, throughput will be non-zero for a relatively larger number of timeslots, but will be smaller. The value $B\left(j, t_{1}, t_{2}\right)$ is obtained by using an optimum value of allocation.

Before giving a formal algorithm to calculate $B\left(j, t_{1}, t_{2}\right)$, we make two observations:

Observation 1: Optimum value of allocation may not be unique. For example, if $c:[2,3,3]$, then using an allocation of 2 , the throughput will be $[2,2,2]$. By using an allocation of 3 , the throughput will be $[0,3,3]$. In each case, the total value of throughput is 6 .

Observation 2: Optimum value of allocation must be equal to the capacity on some timeslot. Otherwise, the net throughput can be achieved by increasing the allocation to next higher capacity.

\subsubsection{Different Rate Allocation within a Sub-carrier Group}

In this case, the value of $B\left(j, t_{1}, t_{2}\right)$ is maximized independently for each channel.

Step 1: Sort the capacities $c(j, t)$, where $t_{1} \leq t \leq t_{2}$ in non-decreasing order. Say the sorted capacities are $c_{1}^{\prime} \leq c_{2}^{\prime} \leq c_{3}^{\prime} \ldots c_{n_{1}}^{\prime}$, where for convenience, we use $n_{1}=t_{2}-t_{1}+1$.

Step 2: If the allocation is equal to $c_{t}^{\prime}$, then the total throughput as given by Equation 1 is $\left(n_{1}-t+1\right) \cdot c_{t}^{\prime}$, because the number of timeslots that have the capacity at least $c_{t}^{\prime}$ is at least $n_{1}-t+1$. 
Step 3: $B\left(j, t_{1}, t_{2}\right)=\max _{t_{1} \leq t \leq t_{2}}\left\{\left(n_{1}-t+1\right) \cdot c_{t}^{\prime}\right\}$.

\section{Time Complexity Analysis}

We observe that Step 1 above can be performed in $O\left(n_{1} \log n_{1}\right)$ time, and Step 3 can be performed in $O\left(n_{1}\right)$ time. Since there are $O\left(n^{2}\right)$ possible values of $t_{1}$ and $t_{2}$, and $O(k)$ possible values for $j$, the entire array $B\left(j, t_{1}, t_{2}\right)$ for all values of $j, t_{1}, t_{2}$ can be calculated on $O\left(k \cdot n^{3} \log n\right)$ time.

\section{An improved $O\left(k \cdot n^{3}\right)$ solution using Dynamic Programming}

The entire array $B\left(j, t_{1}, t_{2}\right)$ can be calculated more effi ciently using dynamic programming.

We observe that given $B\left(j, t_{1}, t_{2}\right)$, the value $B\left(j, t_{1}, t_{2}+1\right)$ can be calculated in $O\left(n_{1}\right)$ time as follows:

We maintain the sorted array of capacities, as well as the frequency count for each index from $t_{1}$ to $t_{2}$. Then, given $c\left(t_{2}+1\right)$, we can insert it into the sorted array in $O\left(n_{1}\right)$ time, update the frequency count in $O\left(n_{1}\right)$ time, and choose the maximum in $O\left(n_{1}\right)$ time. Thus for the $n^{2}$ possible combinations of $t_{1}$ and $t_{2}$, and $k$ possible values of $j$, the complete array $B\left(j, t_{1}, t_{2}\right)$ can be calculated in $O\left(k \cdot n^{3}\right)$ time.

\subsubsection{Same Rate Allocation within a Sub-carrier Group}

In this case, the value of $B\left(G, t_{1}, t_{2}\right)$ is maximized for the entire group at the same time.

Using the approach outlined in Section 3.1.1, the value $B\left(G, t_{1}, t_{2}\right)$ can be calculated by considering all $k n_{1}$ entries at the same time.

Step 1: Sort the capacities $c(j, t)$, where $t_{1} \leq t \leq t_{2}$ and $1 \leq j \leq k$, in non-decreasing order. Say the sorted capacities are $c_{1}^{\prime} \leq c_{2}^{\prime} \leq c_{3}^{\prime} \ldots c_{k n_{1}}^{\prime}$, where for convenience, we use $n_{1}=t_{2}-t_{1}+1$.

Step 2: If the allocation is equal to $c_{t}^{\prime}$, then the total throughput as given by Equation 1 is $\left(k n_{1}-t+1\right) \cdot c_{t}^{\prime}$, because the number of timeslots that have the capacity at least $c_{t}^{\prime}$ is at least $k n_{1}-t+1$.

Step 3: $B\left(G, t_{1}, t_{2}\right)=\max _{t}\left\{\left(k n_{1}-t+1\right) \cdot c_{t}^{\prime}\right\}$.

\section{Time Complexity Analysis}

We observe that assuming $k \leq n_{1}$, Step 1 above can be performed in $O\left(k n_{1} \log n_{1}\right)$ time, and Step 3 can be performed in $O\left(k n_{1}\right)$ time. Since there are $O\left(n^{2}\right)$ possible values of $t_{1}$ and $t_{2}$, the entire array $B\left(G, t_{1}, t_{2}\right)$ for all values of $t_{1}, t_{2}$ can be calculated on $O\left(k \cdot n^{3} \log n\right)$ time.

\section{An improved $O\left(k \cdot n^{3}\right)$ solution using Dynamic Programming}

Similar to Section 3.1.1, the entire array $B\left(G, t_{1}, t_{2}\right)$ can be calculated more effi ciently using dynamic programming 
in this case as well.

We observe that given $B\left(G, t_{1}, t_{2}\right)$, the value $B\left(G, t_{1}, t_{2}+1\right)$ can be calculated in $O\left(n_{1} k\right)$ time as follows: We maintain the sorted array of capacities (in this case, the sorted array has $O\left(n_{1} k\right)$ entries). Then, given $k$ more entries for timeslot $\left(t_{2}+1\right)$, we can fi rst sort the entries in $O(k \log k)$ time. Once we have the sorted entries, we can insert all $k$ entries into the sorted array in a single pass in $O\left(n_{1} k\right)$ time. After that, we can choose the optimum allocation in $O\left(n_{1} k\right)$ time. Thus for the $n^{2}$ possible combinations of $t_{1}$ and $t_{2}$, the complete array $B\left(G, t_{1}, t_{2}\right)$ can be calculated in $O\left(k \cdot n^{3}\right)$ time.

\section{Online Variation of Problem}

In a practical scenario, during timeslot $t$, we do not have the information about what the capacities will be for all timeslots from $t+1$ to $n$. Even with carrier sensing, it is much more reasonable to assume that we have perfect or imperfect information for capacity for the next timeslot, i.e., $(t+1)$ only. Within this realm, many variations of problems are possible, as outlined in Section 2.1 .

\subsection{Online Algorithm given Perfect Estimates for Spectrum and Time}

In this section, we discuss time based perfect estimates and spectrum based perfect estimates, that is, we assume that we have the perfect knowledge for the capacity information of next timeslot $(t+1)$, and by sensing the middle channel in a group, we obtain the perfect information for all channels in that group. For this case, we have the following heuristic algorithm, which is extended from the WDH (wait-dominate-hold) heuristic algorithm presented in authors' earlier work in [8] and [9].

Since we only consider groupwise allocation, we only consider one group $G=\beta_{1}, \beta_{2} \ldots, \beta_{k}$

\subsubsection{Different Allocations Allowed in Same Group}

In this case, our strategy is as follows. For each timeslot $t$, we consider following cases:

1. Use the current timeslot $t$ for link adaptation, that is, set $a(j, t)=0$ for all subchannels $1 \leq j \leq k$. In this case the anticipation is that allocation in the next timeslot, i.e., will be used fully.

That is, we set $\hat{a}(j, t+1)=c(j, t+1)$. In this case, the maximum possible throughput is $\sum_{j=i}^{k} c(j, t+1)$.

2. If the previous timeslot had non-zero allocation, use the current timeslot $t$ without link adaptation, that is, set $a(j, t)=a(j, t-1)>0$ for each subcarrier. 
In this case, the maximum possible throughput is obtained by assuming the same allocation $a(j, t)=\hat{a}(j, t+$ $1)=a(j, t-1)$ on all 3 timeslots. We remind the reader that in this section, we are considering the case in which different channels can use different allocation.

3. If the previous timeslot had zero allocation, use the current timeslot $t$ in the best manner possible with the information that we have.

Based upon the values $c(j, t)$ and $c(j, t+1)$, we consider three subcases:

(a) If $c(j, t) \geq 2 c(j, t+1)$, we set $a(j, t)=\hat{a}(j, t+1)=c(j, t)$. In this case, we note that the throughput in next timeslot, i.e., $x(j, t+1)$ will be 0 .

(b) If $2 c(j, t)<c(j, t+1)$, we set $a(j, t)=\hat{a}(j, t+1)=c(j, t+1)$ and we note that the throughput in the current timeslot, i.e., $x(j, t)$ will be 0 .

(c) If $\frac{1}{2} c(j, t+1)<c(j, t) \leq 2 c(j, t+1)$, we set $a(j, t)=\hat{a}(j, t+1)=\min \{c(j, t), c(j, t+1)\}$. We note that the throughput in both the timeslots will be $x(j, t)=x(j, t+1)=\min \{c(j, t), c(j, t+1)\}$.

We compare the throughput for each case and get the maximum throughput, and allocate corresponding allocations to each channel at timeslot $t$. We calculate the throughput according to the allocation.

\section{Time Complexity Analysis}

As described in the algorithm above, decision for each subcarrier can be made in $O(1)$ time, and thus the time complexity for each timeslot is $O(k)$.

\subsubsection{Same Allocation for all Channels in the Same Group}

In this case, we assume that all channels in a group have same allocation for each timeslot $t$. Since $a(j, t)$ is the same for all subcarriers within a group, we will simply use $a(t)$ to refer to the allocation during the timeslot $t$. That is, $a(t) \stackrel{\text { def }}{=} a(1, t)=a(j, t) \quad 1 \leq j \leq k$.

Suppose that $f(a, t)$ denotes the throughput for the entire group in timeslot $t$, as given by Equation 1 when the base station allocates the data rate of $a$ to that group.

$$
f(a, t)=\sum_{j=1}^{k} x(j, t), \text { when the allocation for all subcarriers is } a
$$




$$
=\sum_{j=1}^{k} a, \text { if } c(j, t) \geq a
$$

Similar to Section 4.1.1, we consider the following cases when deciding allocation for timeslot $t$. We compare the maximum possible throughput for the entire group for two timeslots $t$ and $t+1$ in each case. We then select the maximum throughput, and allocate corresponding allocations to each channel at timeslot $t$.

1. Use the current timeslot for link adaptation, that is, we set $a(t)=0$. In this case, the throughput for the group $G$ during the $t$-th timeslot will be 0 . The tentative throughput for $G$ in timeslot $t+1$ is maximized by choosing a value of allocation (say $\mathrm{a}^{*}$ ) that maximizes $\sum_{j=1}^{k} \hat{x}(j, t+1)$ when the allocation is $a *$. We note that in this case the entire throughput for timeslots $t$ and $t+1$ is tentative, and is given by $f(a *, t+1)$, where $a *=\arg \max \sum_{j=1}^{k} \hat{x}(j, t+1)$.

In this case the optimum value of $a *$ can be calculated by considering all $k$ capacity values for timeslot $t+1$, $a * \in\{c(1, t+1), c(2, t+1), \ldots c(k, t+1)\}$.

2. If the previous timeslot allocation was larger than 0 , and we continue the same allocation, that is, we set $a(t)=$ $a(t-1)>0$. In this case, the maximum possible throughput is obtained by retaining the same throughput in timeslot $t+1$ as well, that is by setting $a(t+1)=a(t-1)$, and the throughput is $f(a *, t)+f(a *, t+1)$, where $a *=a(t-1)$.

3. If the previous timeslot allocation was 0 , and we choose new allocation $a(t)=a(t+1)>0$. In this sub-case, the maximum possible throughput is:

$f(a *, t)+f(a *, t+1)$, where $a *=\arg \max \sum_{1 \leq j \leq k}\{x(j, t)+\hat{x}(j, t+1)\}$.

In this case the optimum value of $a *$ can be calculated by considering all $2 k$ capacity values for timeslot $t+1$, $a * \in\{c(1, t+1), c(2, t+1), \ldots c(k, t+1), c(k+1, t+1), c(k+2, t+1), \ldots c(2 k, t+1)\}$.

\section{Time Complexity Analysis}

From the algorithm description above, the worst case time is for fi nding the optimum allocation value is in Case 3. In that case, given $2 k$ possible values of $a *$, the optimum value can be computed by fi rst sorting all possible values, similar to as described in Section 3.1.2. Thus, the time complexity for each timeslot is $O(k \log k)$. 


\subsection{Heuristic for Imperfect Spectrum Estimates}

In this subsection, we consider the case in which we only have the perfect knowledge of the center channel in a group $G_{s}$, i.e., $c_{s}^{*}(i, t)=c\left(i^{\prime}, t\right)$ and $c_{s}^{*}(i, t+1)=c^{\prime}\left(i^{\prime}, t+1\right)$, where $\beta_{i^{\prime}}$ is the center of $G_{s}$. We assume that the possible data rates are limited, which are denoted by $R=\left\{r_{1}, \ldots, r_{M}\right\}$. We also assume that $Q_{s}(a, b, i, t)$ is given for any $(a, b)$ pair, where $a$ and $b$ are data rates included in $R$. We estimate the $c(i, t)$ with $c_{e}(i, t)=\sum_{q=1}^{M} Q_{s}\left(c_{j}^{*}(i, t), r_{q}, i, t\right)$.

We also estimate $c^{\prime}(i, t+1)$ with $c_{e}^{\prime}(i, t+1)=\sum_{q=1}^{M} Q_{s}\left(c_{j}^{*}(i, t+1), r_{q}, i, t\right)$. Note that $c_{e}(i, t)$ and $c_{e}^{\prime}(i, t+1)$ are expected values. After that, the base station can apply heuristic for perfect spectrum estimates to make allocation.

\section{Numerical Results}

In this section, we present the empirical results for the algorithms presented in the preceding sections. Our goal here is to explore the impact on the throughput and optimal throughput with variant group size for different cases discussed in the previous sections.

For calculating empirical results, we assume 1,000 timeslots and channel capacity is randomly chosen from one of the 9 levels (MCS: $[8.8,11.2,14.8,17.6,22.4,29.6,44.8,54.5,59.2]($ in $\mathrm{kb} / \mathrm{s})$ ) as outlined in the EGPRS modulation and coding schemes and peak data rates in [1].

For the case of algorithms for time based imperfect channel estimates, the following procedure is used to calculate the estimated capacity and true capacity values. The prediction accuracy is used to calculate the values $P(a, b)$ for all values of $b \neq a$, where $P(a, b)$ is the probability that actual capacity $c(j, t+1)$ turns out to be equal to $b$, given the estimated value $c^{\prime}(j, t+1)$ was equal to $a$.

1. We assume that for any rate $a$, the prediction accuracy in capacity estimation for each channel, and each time slot is the same, and is given by $P(a, a)$.

2. We fi rst generate the value $a$ by choosing a random value from the MCS list.

3. From $P(a, a)$, we fi rst calculate $P(a, \bar{a})=1-P(a, a)$.

4. We assume that the $P(a, b)$ is inversely proportional to the "distance" between $a$ and $b$. Please note that the distance is between the indices in the MCS list. For example, the distance between 11.2 and 22.4 is 3 . Using this inverse relationship and the fact that $\sum_{b \in \mathrm{MCS}} P(a, b)=1$, we can calculate $P(a, b)$ for all values of $a$ and $b$. 
We note that many results for the case of perfect estimation can be subsumed under the results for imperfect estimation, by setting the prediction accuracy probability to 1 .

For the case of algorithms for spectrum based imperfect channel estimates, the following procedure is used to calculate the estimated capacity and true capacity values. The prediction accuracy is used to calculate the values $Q(a, b)$ for all values of $b \neq a$, where $Q(a, b)$ is the probability that actual capacity $c(i, t)$ turns out to be equal to $b$, given the estimated value $c_{j}^{*}(i, t)$ was equal to $a$.

1. We assume that for any rate $a$, the prediction accuracy in capacity estimation for each time slot is the same, and only related with the "distance" which is given by $Q_{j}(a, a, i)$. Please note that the distance is between the indices of $\beta_{i}$ and $\beta_{j}$, where $\beta_{j}$ is the center channel of the group.

2. We fi rst generate the value $a$ by choosing a random value from the MCS list, which will apply to all channels in a group.

3. From $Q_{j}(a, a, i)$, we fi rst calculate $Q_{j}(a, \bar{a}, i)=1-Q_{j}(a, a, i)$.

4. We assume that the $Q_{j}(a, b, i)$ is inversely proportional to the "distance" between $a$ and $b$. Please note that the distance is between the indices in the MCS list. For example, the distance between 11.2 and 22.4 is 3 . Using this inverse relationship and the fact that $\sum_{b \in \operatorname{MCS}} p(a, b)=1$, we can calculate $Q_{j}(a, b, i)$ for all values of $a$ and $b$.

We note that many results for the case of time based perfect estimation can be subsumed under the results for time based imperfect estimation, by setting the prediction accuracy probability to 1.0 .

Next we plot throughput for different group size, different values of prediction accuracy, as well as the two allocation schemes (same or different allocation in each timeslot). The number of channels considered is 30 . The data, which stand for capacities for each timeslot and each channel, are generated randomly in Figures 1, 2 and 3.

In Figure 1, the throughput is plotted against the group size, for prediction accuracy 0.6, 0.8 and 1.0 with monitoring all channels in a group, i.e., the capacity estimate of each channel in timeslot $t$ has $100 \%$ accuracy. The plot suggests that the throughput decreases with the increasing group size, and the scheme with different rate allocation within a group always results in higher throughput than the scheme with same allocation. The results also confi rm that the throughput is higher if the time-based prediction accuracy for the channel is higher. This is also consistent for results shown in Figures 2 and 3. 


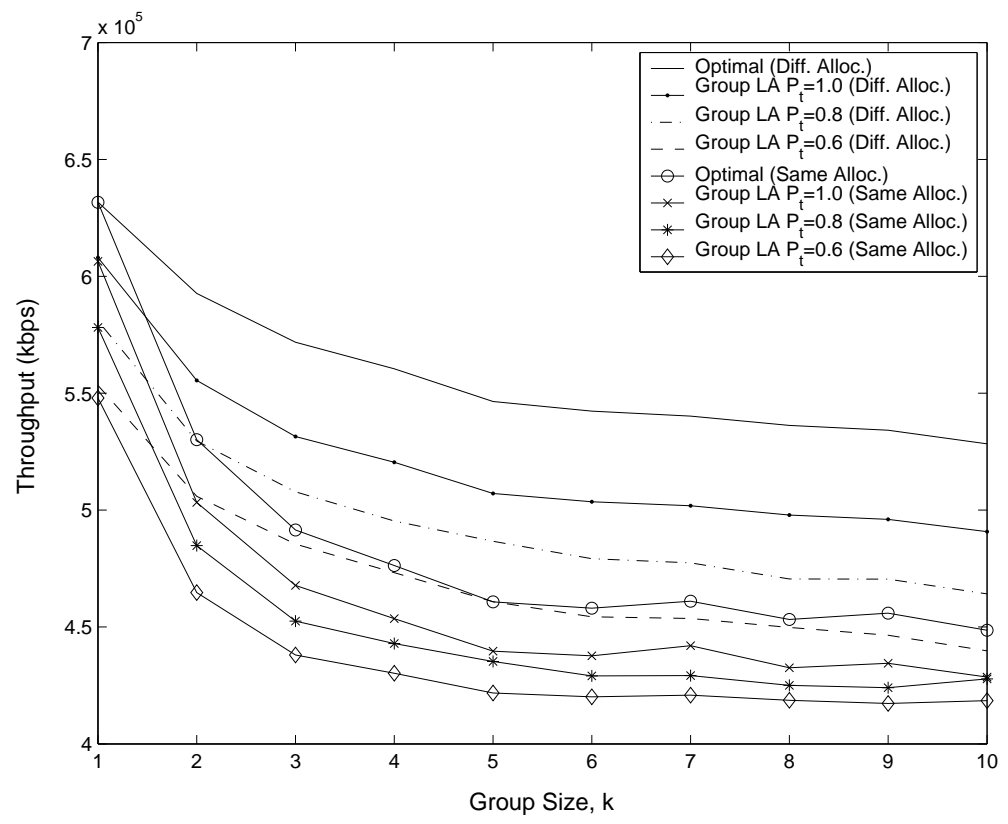

Figure 1: Throughput vs. Group Size for spectrum based perfect estimates, $f=30, P_{t}$ is the accuracy of estimated capacity for next timeslot.

In Figures 2 and 3, results are shown for the case when only the channel located in the center is monitored, and the throughput is plotted against the group size, for $P_{s}=0.1$ and $P_{s}=0.2$, respectively, and for the prediction accuracy 0.6, 0.8 and 1.0, where $P_{s}$ is the parameter to calculate $Q_{j}(a, a, i)=1-P_{s} d$ and here $d$ is the distance between $\beta_{j}$ and $\beta_{i}$. The plots suggest that for each case, the throughput is less than that in Figure 1. However, the throughput for each case shows a similar trend when the group size increases. This can be explained due to the fact that the capacity of each channel in Figure 1 has 100\% accuracy, while in Figures 2 and 3, the base station only has the perfect knowledge for the channel residing in the center of group.

Note that channel capacities are randomly generated in Figures 2 and 3, while heuristic algorithms consider the different values of $Q_{j}(a, a, i)$. Therefore, one cannot expect the outcome of each case, i.e., the throughput when $P_{s}=0.1$ is not necessarily better than the throughput when $P_{s}=0.2$. In Figure 4, we generated capacities following the same method for calculating $Q_{j}(a, a, i)$ and compared the result when the same allocation constraint is applied. As expected, the throughput for the case of $P_{s}=0.1$ always performs better than for the case of $P_{s}=0.2$. 


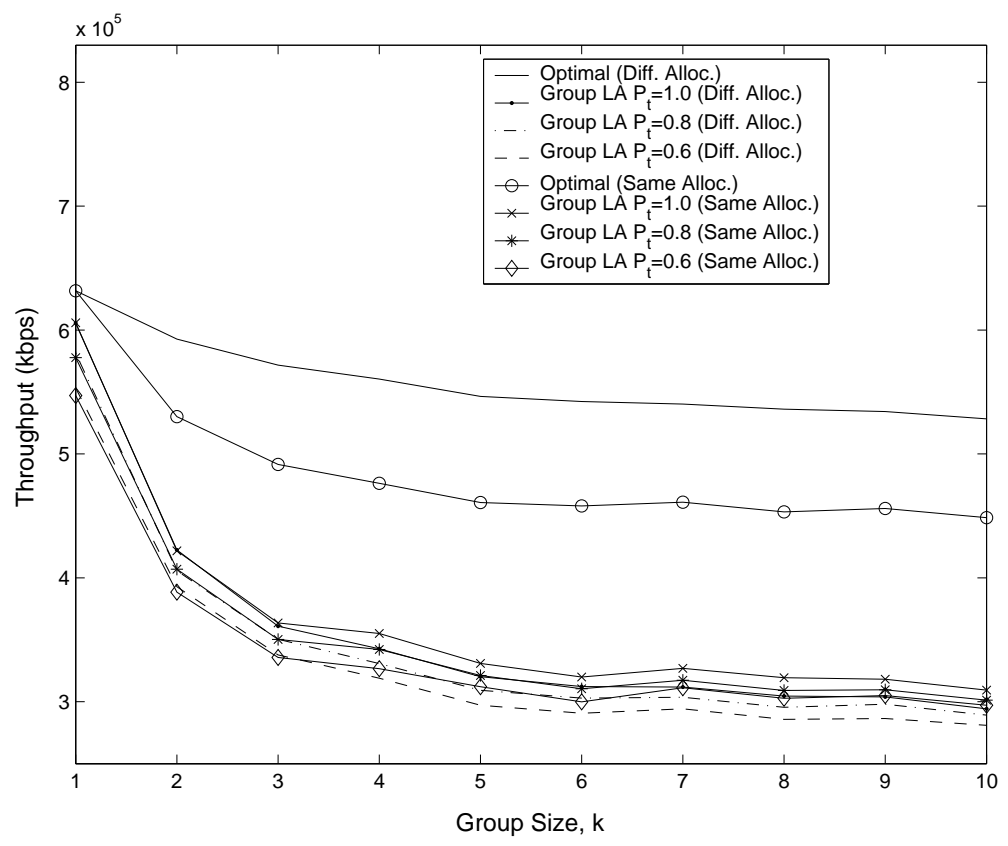

Figure 2: Throughput vs. Group Size assuming that $P_{s}=0.1, f=30, P_{t}$ is the accuracy of estimated capacity for next timeslot.

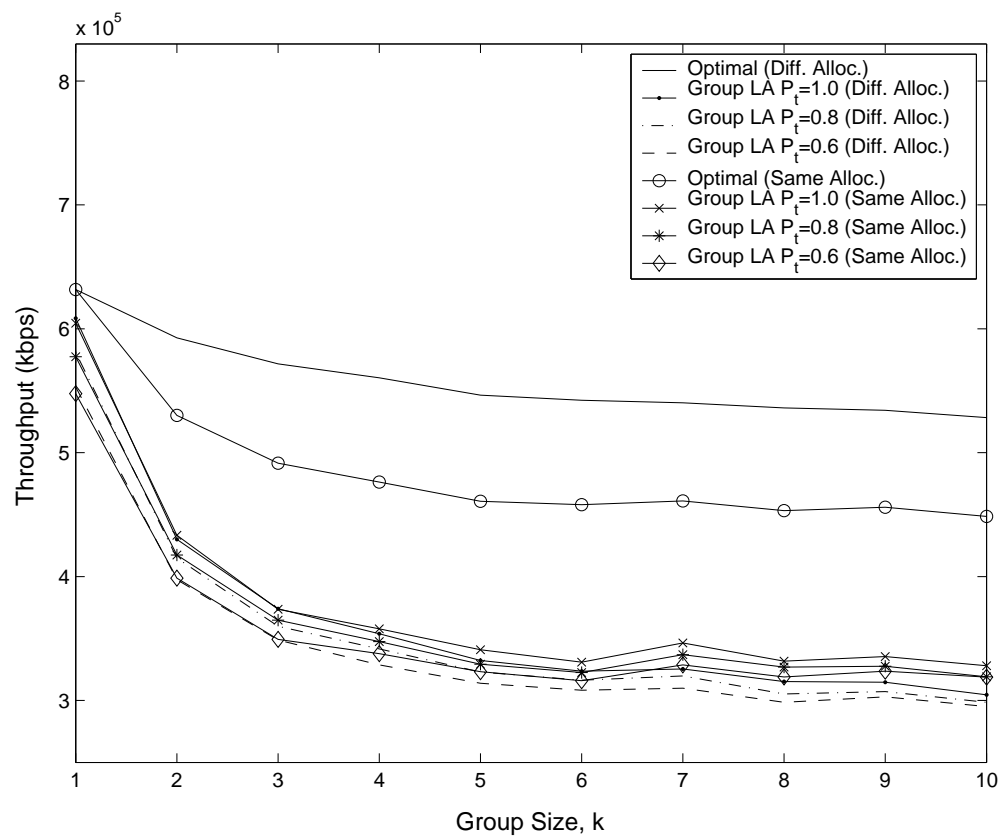

Figure 3: Throughput vs. Group Size assuming that $P_{s}=0.2, f=30, P_{t}$ is the accuracy of estimated capacity for next timeslot. 


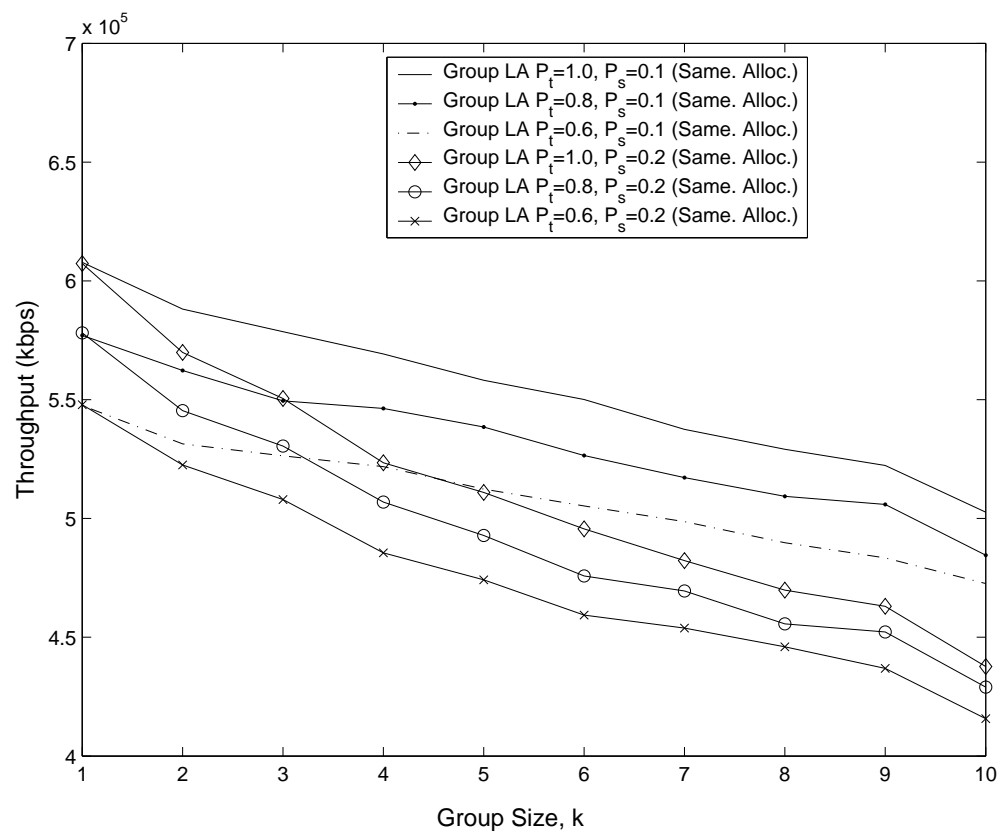

Figure 4: Throughput vs. Group Size for two cases of $P_{s}=0.1$ and $P_{s}=0.2$ when same allocation constraint is applied, , $f=30, P_{t}$ is the accuracy of estimated capacity for next timeslot.

\section{Conclusion}

Sub-carrier grouping is a promising mechanism for making link adaptation in multi-carrier networks practical. In this paper, we have considered the throughput maximization problem over a multi-carrier link for various sub-carrier grouping models, each corresponding to a different level of resource consumption for LA, and implementation complexity. These models varied based on whether (i) the CSI for each sub-carrier within a group is monitored individually or not (i.e., perfect or imperfect estimates over spectrum), and (ii) all sub-carriers within the group are required to use the same data rate (or mode) or not. In addition, we have also considered the cases with imperfect estimates over time. We have presented a dynamic programming algorithm for obtaining the off-line optimal solution for the perfect spectrum and time estimates case, for both the model that uses the same rate within a group, and the one that does not. We proposed an online scheduling algorithm for the same case, which uses only a single time-slot look-ahead. Simulations, and the optimal solution obtained through dynamic programming to the off-line solution were used to demonstrate the near-optimal performance of the proposed online algorithm. We then proposed heuristics based on this online algorithm that extend to the cases with imperfect estimates over time and spectrum. We have also studied the trade-offs between the group size and the throughput for each model. As expected, the throughput decreases with 
increasing group size, although the additional throughput penalty for increasing the group size is observed to be less for large group sizes. We also observed that whether the sub-carriers within the same group are required to use the same mode or not has an important effect on the throughput. For example, assuming perfect spectrum and time-based estimates, about $30 \%$ higher throughput can be achieved by allowing the use of different modes. Overall, our results can be used in assessing different sub-carrier grouping architectures and grouping sizes in terms of their effects on the throughput.

\section{References}

[1] S. Catreux, V. Erceg, D. Gesbert, and R.W. Heath, "Adaptive modulation and MIMO coding for broadband wireless data networks,” IEEE Communications Magazine, vol. 40, pp. 108-115, June 2002.

[2] V. Tsibonis, L. Georgiadis, and L. Tassiulas, "Exploiting wireless channel state information for throughput maximization,' in Proceedings of IEEE Infocom '03, April 2003, pp. 301-310.

[3] S. Kulkarni and C. Rosenberg, "Opportunistic scheduling policies for wireless systems with short term fairness constraints," in Proceedings of Globecom 2003, December 1992, pp. 533-537.

[4] X. Liu, E.K.P. Chong, and N. Shroff, "Opportunistic transmission scheduling with resource-sharing constraints in wireless networks," IEEE JSAC, vol. 19, no. 10, pp. 2053-2064, Oct. 2001.

[5] T. Keller and L. Hanzo, "Adaptive multicarrier modulation: a convenient framework for time-frequency processing in wireless communications," Proceedings of the IEEE, vol. 88, pp. 1369-1394, August 1990.

[6] J.E. Kleider and S. Gifford, "Synchronization for broadband OFDM mobile ad hoc networking: simulation and implementation," in Proceedings of IEEE ICASSP 2002, May 2002, pp. IV3756-IV3759.

[7] S. Armour, A. Nix, and D. Bull, "Complexity evaluation for the implementation of a pre-FFT equalizer in an OFDM receiver," IEEE Transactions on on Consumer Electronics, vol. 46, no. 3, pp. 428-437, 2000.

[8] G. Sahin, F. Jin, A. Arora, and H.A. Choi, "Trade-offs between prediction quality and throughput in wireless network scheduling with link adaptation," in IEEE Global Telecommunications Conference, 2004, Submitted.

[9] G. Sahin, F. Jin, A. Arora, and H.A. Choi, "Predictive scheduling in multi-carrier wireless networks with link adaptation," in IEEE Vehicular Technology Conference, 2004, Submitted. 